\title{
Solar Parabolocilindrical Installations, Constructive Features and Calculation of Individual Parameters
}

\author{
Sirozhiddin F. Ergashev and Ulugbek Zh. Nigmatov* \\ Ferghana Polytechnic Institute \\ Ferghana, Republic of Uzbekistan
}

Received 12.01.2021, received in revised form 09.02.2021, accepted 16.02.2021

\begin{abstract}
The article gives a brief description of a solar parabolic-cylindrical installation and its main element, an optical concentrator. The analysis is based on the most simplified and sufficiently accurate methodology for calculating the coefficient of geometric concentration of parabolic-cylindrical mirrors. The cases of an ideal parabolic-cylindrical concentrator having a small aperture are considered. A variant of calculating the concentration coefficient of a parabolic cylindrical concentrator for cylindrical and flat receivers is presented.
\end{abstract}

Keywords: parabolic cylinder, concentrator, geometric, aperture, receiver, focusing, elliptical.

Citation: Ergashev S.F., Nigmatov U.Zh. Solar parabolocilindrical installations, constructive features and calculation of individual parameters, J. Sib. Fed. Univ. Eng. \& Technol., 2021, 14(1), 33-41. DOI: 10.17516/1999-494X-0286

\section{Солнечные параболоцилиндрические установки, конструктивные особенности \\ и расчет отдельных параметров}

\author{
С. Ф. Ергашев, У.Ж. Нигматов \\ Ферганский политехнический институт \\ Республика Узбекистан, Фергана
}

Аннотация. В статье дана краткая характеристика солнечной параболоцилиндрической установки и его основного элемента - оптического концентратора. Произведен анализ на основе наиболее упрощенной и достаточно точной методики расчета коэффициента геометрической концентрации параболоцилиндрических зеркал. Рассмотрены случаи идеального параболоцилиндрического

(C) Siberian Federal University. All rights reserved

This work is licensed under a Creative Commons Attribution-Non Commercial 4.0 International License (CC BY-NC 4.0).

* Corresponding author E-mail address: fesirojiddin@bk.ru,ferpi_info@edu.uz 
концентратора, имеющего небольшую апертуру. Представлен вариант вычисления коэффициента концентрации параболоцилиндрического концентратора для цилиндрических и плоских приемников.

Ключевые слова: параболоцилиндр, концентратор, геометрический, апертура, приемник, фокусирование, эллиптический.

Цитирование: Ергашев, С.Ф. Солнечные параболоцилиндрические установки, конструктивные особенности и расчет отдельных параметров / С.Ф. Ергашев, У.Ж. Нигматов // Журн. Сиб. федер. ун-та. Техника и технологии, 2021, 14(1). C. 33-41. DOI: $10.17516 / 1999-494 X-0286$

\section{Введение}

В настоящее время большое внимание уделяется разработке, экспериментальному исследованию и практическому применению солнечных параболоцилиндрических энергетических установок как одного из наиболее дешевых и перспективных вариантов преобразования солнечной энергии в электрическую и тепловую [1-4]. Это связано с тем, что в параболоцилиндрических концентраторах регулировка и фокусировка зеркал осуществляются с относительно невысокой точностью и легко $[5,6]$. Они могут работать с простыми системами слежения за Солнцем. Вследствие этого в целом капитальные затраты на эти установки могут быть небольшими [7].

Зеркальная поверхность оптического концентратора образуется продольным передвижением параболы по длине оси, перпендикулярной к оси, проходящей через ее вершину. Поэтому параболоцилиндрическими называются зеркала, имеющие вид корыта (желоба) с профильным сечением в форме параболы, а в продольном сечении - в виде прямой плоскости. В отличие от параболоидных параболоцилиндрические концентраторы не создают максимальную концентрацию лучистого потока, так как они в профильной плоскости работают аналогично параболоидному отражателю, а в продольной - плоскому зеркалу. Следовательно, параболоцилиндрические установки относят к среднетемпературным солнечным установкам. Диапазон их рабочих температур $60 \div 460{ }^{\circ} \mathrm{C}[8]$.

Следует отметить, что конструктивно и технологично удобно выполнять параболоцилиндрические установки по модульной схеме, когда каждый модуль представляет собой оптический концентратор с приемником тепла и системой слежения.

Исполнение по модульной схеме позволяет собирать установку любой необходимой мощности, а также существенно упрощать опорные конструкции и механизмы привода системы слежения за Солнцем. Систематизирование методов расчета и технологии изготовления, а по конструктивным признакам выявление конструктивных и технологических достоинств и недостатков каждого элемента параболоцилиндрической системы, а также сравнительная оценка их энергетических и технико-экономических характеристик актуальны и представляют определенный практический интерес.

\section{Методы и материалы}

Проанализируем наиболее упрощенную и достаточно точную методику расчета коэффициента геометрической концентрации параболоцилиндрических зеркал. Вопросы расчета коэффициента геометрической концентрации $K_{\Gamma}$ параболоцилиндрических зеркал рассма-

$$
-34-
$$


тривались многими авторами [9-12]. Были предложены простые выражения для определения среднегеометрической и максимальной величины коэффициента геометрической концентрации. Кроме того, решались задачи выбора оптимальных размеров и формы приемника для достижения максимальной степени концентрации.

В случае идеального параболоцилиндрического концентратора, имеющего небольшую апертуру (рис. 1), степень геометрической концентрации определяется выражением

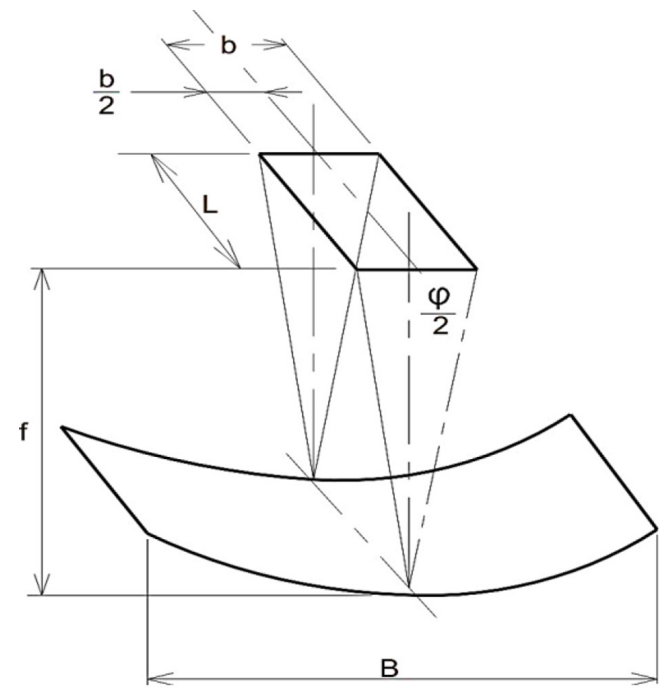

Рис. 1. Схема параболоцилиндрического концентратора с небольшой апертурой $B$

Fig. 1. Diagram of a parabolic cylindrical concentrator with a small aperture $B$

$$
K_{\Gamma}=\frac{B L}{b L}=\frac{B}{b} .
$$

Известно, что с увеличением апертуры увеличивается и фокусное расстояние, а также угол раскрытия (охвата) концентратора. Эта связь (так называемая степень раскрытия зеркала) характеризуется уравнением

$$
\frac{B}{f}=n
$$

на рис. 1 видно, что ширина фокального пятна $b$ определяется выражением

$$
b=2 f \operatorname{tg} \frac{\varphi}{2}
$$

подставляя выражения (2) и (3) в уравнение (1), получаем

$$
K_{\Gamma}=\frac{n}{2 \operatorname{tg} \varphi / 2}
$$

с учетом того, что для идеального концентратора $\varphi / 2=16^{\prime}$ или 0,00467 рад, уравнение (4) имеет вид $[9,11]$

$$
K_{\Gamma}=107,3 \mathrm{n} .
$$

Наиболее оптимальная аппроксимация данного уравнения осуществляется при $\mathrm{n}<5$ и $\mathrm{n} \rightarrow 0$.

$$
-35-
$$




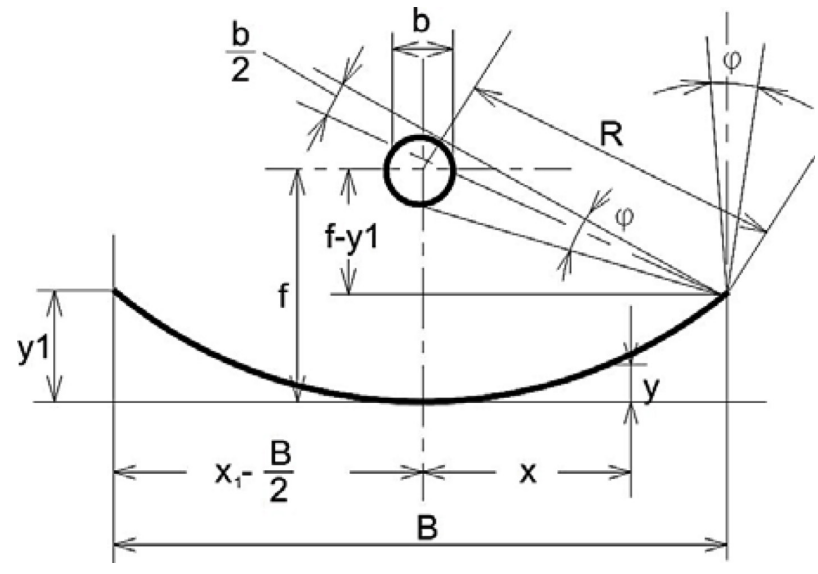

Рис. 2. Схема параболоцилиндрического концентратора с цилиндрическим приемником

Fig. 2. Diagram of a parabolic-cylindrical concentrator with a cylindrical receiver

Вычислим коэффициент геометрической концентрации К Г параболоцилиндрического концентратора для цилиндрических и плоских приемников.

Для цилиндрического приемника (рис. 2) коэффициент геометрической концентрации определяется формулой

$$
K_{\Gamma}=\frac{B L}{\pi d L}=\frac{B}{\pi d}
$$

диаметр приемника находится из выражения

$$
\sin \frac{\varphi}{2}=\frac{d / 2}{R}=\frac{d}{2 R}
$$

откуда

$$
d=b=R(2 \sin \varphi / 2) ;
$$

где $R$ - радиус отражающей точки относительно фокуса.

На рис. 2 видно, что радиус $R$ выражается уравнением

$$
R=\sqrt{\left(f-y_{1}\right)+x_{1}^{2}}
$$

согласно свойству параболы,

$$
x^{2}=4 f y
$$

и, следовательно,

$$
R=\sqrt{\left(f-y_{1}\right)^{2}+4 f y_{1}}=\sqrt{\left(f+y_{1}\right)^{2}}=f+y_{1} ;
$$

однако

$$
y_{1}=x_{1}^{2} / 4 f
$$

и

$$
n=\frac{B}{f}=\frac{2 x_{1}}{f}
$$


или

$$
x_{1}=\frac{n f}{2}
$$

с учетом уравнений (6) - (12) и (13) выражение коэффициента К Г для цилиндрического приемника имеет вид

$$
K_{\Gamma}=\frac{B}{\pi d}=\frac{n f}{\pi\left[R\left(2 \sin \frac{\varphi}{2}\right)\right]}=\frac{n f}{\pi\left[f\left(1+\frac{n^{2}}{16}\right)\left(2 \sin \frac{\varphi}{2}\right)\right]}=\frac{n}{\pi\left(1+\frac{n^{2}}{16}\right)\left(2 \sin \frac{\varphi}{2}\right)} \cong \frac{107,3 n}{\pi\left(1+\frac{n^{2}}{16}\right)}
$$

Продифференцировав уравнение (15) по $n$, можно вычислить максимальную степень концентрации $K_{\Gamma}^{\max }=68,2$ для цилиндрического приемника [9].

Рассмотрим коэффициент концентрации параболоцилиндрического концентратора для плоского приемника (рис. 3):

$$
K_{\Gamma}=\frac{B L}{b L}=\frac{B}{b} ;
$$

На рис. 3 видно, что ширина приемника b равна

$$
b=2 b_{1}
$$

$\mathrm{a}_{1}$, из $\triangle \mathrm{ABC}-$

$$
\frac{b_{1}}{\sin \gamma}=\frac{b_{1}^{\prime} \sin \alpha}{\sin \delta}
$$

следовательно,

$$
b_{1}=\frac{b_{1}^{\prime} \sin \gamma}{2 \sin \delta}
$$

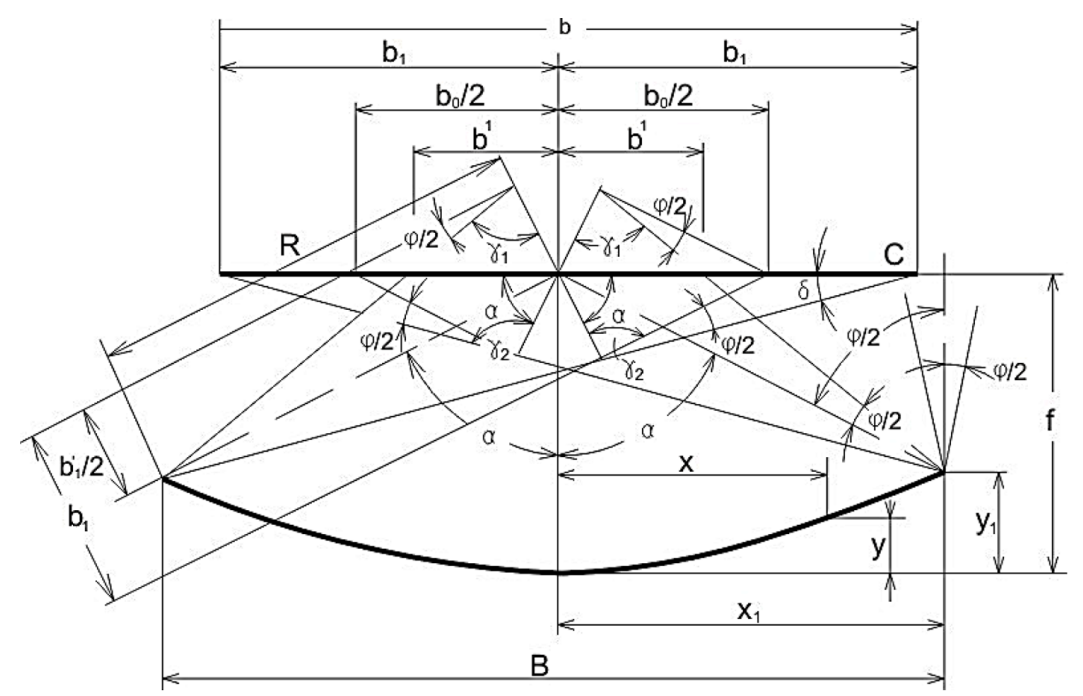

Рис. 3. Расчетная схема параболоцилиндрического концентратора с плоским приемником [9]

Fig. 3. Calculation diagram of a parabolic-cylindrical concentrator with a flat receiver [9]

$$
-37-
$$


с учетом того, что

$$
\gamma=90^{\circ}+\frac{\varphi}{2}
$$

выражение (19) принимает вид

$$
\delta=180^{\circ}-\gamma-\alpha=90^{\circ}-\frac{\varphi}{2}-\alpha
$$

однако для $\varphi=32^{\prime}$

$$
\begin{aligned}
& \cos \frac{\varphi}{2}=1,00, \\
& \sin \frac{\varphi}{2}=0,00467,
\end{aligned}
$$

с учетом (22) и (23) уравнение (21) принимает вид

$$
b_{1}=b_{1}^{\prime} / 2 \cos \alpha
$$

величина $b_{1}^{\prime}$ (рис. 3) определяется формулой

$$
b_{1}^{\prime}=R\left(2 \operatorname{tg} \frac{\varphi}{2}\right) f\left(1+\frac{n^{2}}{16}\right)\left(2 \operatorname{tg} \frac{\varphi}{2}\right)
$$

угол охвата $\alpha$ (рис. 3) выражается формулой

$$
\cos \alpha=\frac{f-y}{R}
$$

или

$$
\cos \alpha_{1}=\frac{f-y_{1}}{R_{1}}=\frac{f-y_{1}}{f+y_{1}}
$$

Используя соотношения (27) и (26), уравнение (25) можно записать в следующем виде:

$$
\cos \alpha_{1}=\frac{f-\frac{n^{2} f}{16}}{f+\frac{n^{2} f}{16}}=\frac{1-\frac{n^{2}}{16}}{1+\frac{n^{2}}{16}}
$$

и, наконец, ширина приемника определяется по формуле

$$
b=\frac{b_{1}^{\prime}}{\cos \alpha} \cong \frac{f\left(1+\frac{n^{2}}{16}\right)^{2}\left(2 \operatorname{tg} \frac{\varphi}{2}\right)}{\left(1+\frac{n^{2}}{16}\right)}
$$

подставив выражения (29) и (28) в уравнение (15), получим

$$
K_{\Gamma}=\frac{n f}{f\left(1+\frac{n^{2}}{16}\right)^{2}\left(2 \operatorname{tg} \frac{\varphi}{2}\right)}=\frac{107,3 n\left(1-\frac{n^{2}}{16}\right)}{\left(1+\frac{n^{2}}{16}\right)^{2}}
$$

Дифференцируя выражение (30) по $n$, можно вычислить максимальную степень концентрации $K_{\Gamma}^{\max }=107,3$ для плоского приемника $[9,13,14]$.

$$
-38-
$$




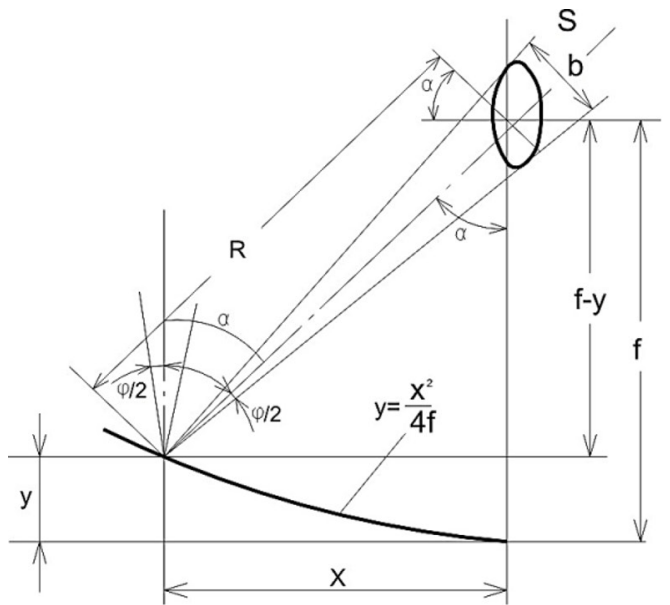

a)

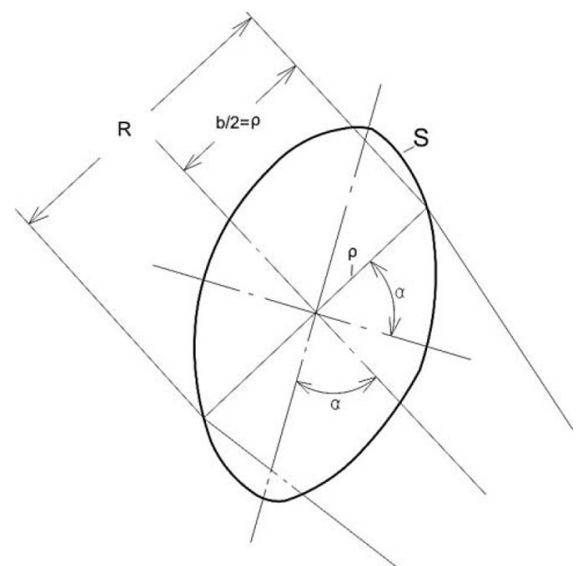

б)

Рис. 4. Расчетная схема концентратора (а) и оптимальная форма приемника (б), имеющая сечение эллиптического цилиндра [9]

Fig. 4. Calculation diagram for the concentrator (a) and the optimal shape of the receiver (b), having a crosssection elliptical cylinder [9]

Для параболоцилиндрического концентратора оптимальной является форма приемника, имеющего сечение эллиптического цилиндра (рис. 4).

Коэффициент геометрической концентрации для приемника, имеющего форму эллиптического цилиндра, выражается формулой

$$
K_{\Gamma}=\frac{B L}{S L}=\frac{B}{S},
$$

где $S$ - длина окружности эллиптического цилиндра.

С учетом рис. 3 и рис. 4 , а также выражений, приведенных выше для $S$, M.H. Cobble [1] получил соотношение

$$
S \approx 4,598 f\left(2 \operatorname{tg} \frac{\varphi}{2}\right) .
$$

Подставив (31) в (32) для К Г, получим

или

$$
K_{\Gamma}=\frac{n f}{4,598 f\left(2 \operatorname{tg} \frac{\varphi}{2}\right)}=\frac{n}{4,598\left(2 \operatorname{tg} \frac{\varphi}{2}\right)}
$$

$$
K_{\Gamma}=\frac{107,3 n}{4,598}=23,34 n \text {. }
$$

Для параболоцилиндрического концентратора, имеющего степень раскрытия $\mathrm{n}=4$, величина $K_{\Gamma}^{\text {onm. }}=93,8$, что по сравнению с цилиндрическим приемником, имеющим $K_{\Gamma}^{\max }=68,3$ при $\mathrm{n}=4$, является существенным. Однако реализовать эффективность эллиптических приемников на практике невозможно (трудно технологически), так как промышленность выпускает только цилиндрические трубы.

Приведенный выше аналитический расчет геометрической концентрации параболоцилиндрических концентраторов с приемниками различной формы показывает, что геометрическая 


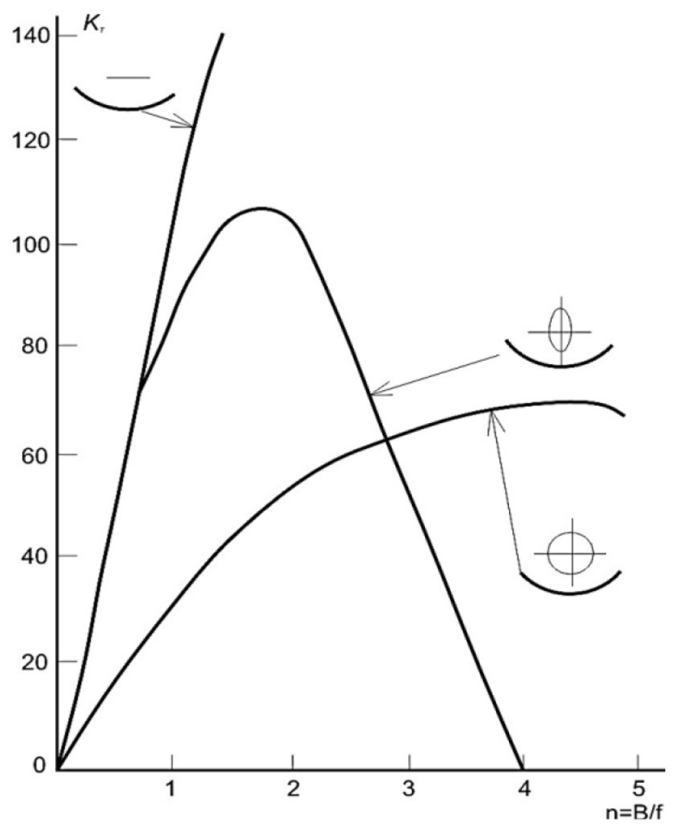

Рис. 5. Зависимость коэффициента концентрации параболоцилиндрического концентратора КГ в функциях степени раскрытия для различных форм приемников [9]

Fig. 5. The concentration factor dependence for the parabolic-cylindrical concentrator KG in functions of the opening degree for various receivers' forms [9]

концентрация К Г параболоцилиндрического концентратора с приемником в форме цилиндрической трубы равна 34,2 n, а с плоским приемником - 107,3 n, которые можно использовать в инженерных расчетах солнечных энергетических установок.

\section{Выводы}

Систематизированы и установлены расчетные выражения для определения оптикогеометрических, точностных и энергетических параметров параболоцилиндрического концентратора: распределения отраженного потока лучистой энергии в фокальной плоскости Er; коэффициента средней энергетической $\mathrm{K}_{\ni}^{\mathrm{cp}}$ и геометрической концентрации $\mathrm{K}_{\Gamma}$; параметр (мера) точности $\mathrm{h}$; расчетный угол раскрытия отраженного пучка $\varphi$; угловая характеристика неточности зеркала $\Delta \varphi$.

\section{Список литературы / References}

[1] Kalogirou, S. A. Solar Energy Engineering-Processes and Systems, 2nd ed., Elsevier. 2014. $762 \mathrm{p}$.

[2] Roman Bader, Andrea Pedretti, Aldo Steinfeld. A 9-m-Aperture Solar Parabolic Trough Concentrator Based on a Multilayer Polymer Mirror Membrane Mounted on a Concrete Structure. Journal of Solar Energy Engineering, 2011, 133, 12-16.

[3] Avezova N.R., Khaitmukhamedov A. E., Usmanov A. Yu., and Boliyev B. B. Solar Thermal Power Plants in the World: The Experience of Development and Operation, Applied Solar Energy, 2017, 53, 1, 72-77, doi: 10.3103/S0003701X17010030.

$$
-40-
$$


[4] Klychev S.I., Abdurakhmanov A.A., Kuchkarov A. A. Optical-geometric parameters of a linear Fresnel mirror with flat facets, Applied Solar Energy, 2014, 50, 168-170, doi.org/ 10.3103/ S0003701X14030074.

[5] Lovegrove K., Stein W. Concentrating solar power technology 1st Edition (Principles, developments and applications), Woodhead Publishing Series in Energy, 2012, 21, 704. https://www. elsevier.com/books/concentrating-solar-power-technology/lovegrove/978-1-84569-769-3.

[6] Kuchkarov A.A. et al. Calculation of Thermal and Exergy Efficiency of Solar Power Units with Linear Radiation Concentrator. Applied Solar Energy, 2020, 56(1), 42-46.

[7] Klychev, Sh.I., Modeling of receiving - concentrating devices of solar thermal power units, Doctoral (Tech.Sci.) Dissertation, Tashkent, FTI, 2004.

[8] Mukhitdinov, M.M. and Ergashev, S.F. Solnechnye parabolotsilindricheskie ustanovki (Solar Parabolic Cylinders), Tashkent: FAN, 1995.

[9] Cobble M. H. Theoretical concentrations for solar furnaces. Solar Energy, 1961, 5(61), 72.

[10] Klychev Sh.I., Zakhidov R.A., Bakhramov S.A., Fasylov A.K., Dudko Yu.A. Solar Radiation Concentration in Parabolocylindrical System with Focusing Wedge. Applied Solar Energy, 2009,45, 2, 99-101.

[11] Kuchkarov A.A., Abdurakhmanov A.A., Mamatkosimov M.A., Akhadov Zh. The optimization of the optical-geometric characteristics of mirror concentrating systems. Applied Solar Energy, 2014, 50, 244-251.

[12] Fernandez-Garcia, A., Zarza, E., Valenzuela, L., andPerez, M., Parabolic-trough solar collectors and their applications, Renewable Sustainable Energy Rev., 2010, 14(7), 1695-1721.

[13] Kuchkarov, A.A., Kholova, Sh.R., Abdumuminov, A.A., and Abdurakhmanov, A., Optical energy characteristics of the optimal module of a solar composite paraboliccylindrical plant, Appl. Sol. Energy, 2018, 54(4), 293-296.

[14] Fei Chen, Ming Li, Peng Zhang. Distribution of Energy Density and Optimization on the Surface of the Receiver for Parabolic Trough Solar Concentrator, International Journal of Photoenergy, 2015. Article ID120917. P. 10. 